\title{
The novel c-Met inhibitor cabozantinib overcomes gemcitabine resistance and stem cell signaling in pancreatic cancer
}

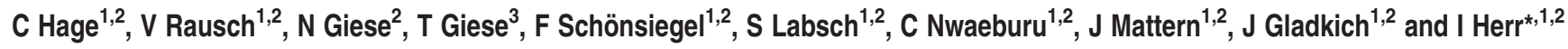

Pancreatic ductal adenocarcinoma (PDA) is one of the most lethal malignancies. Cancer stem cells (CSCs), which are not targeted by current therapies, may be the reason for pronounced therapy resistance. A new treatment option in phase II trials is cabozantinib that inhibits the pancreatic CSC surface marker and tyrosine kinase receptor c-Met. The purpose of this study was to evaluate the effect of cabozantinib to stem-like features and therapy resistance. Established PDA cell lines, a gemcitabine-resistant subclone, non-malignant pancreatic ductal cells and primary spheroidal cultures from patient tumors were analyzed by MTT-assay, flow cytometry, colony and spheroid formation assays, western blotting, qRT-PCR, antibody protein array, immunohistochemistry and morphological features. Cabozantinib inhibited viability and spheroid formation and induced apoptosis in malignant cells with minor effects in non-malignant cells. After long-term cabozantinib treatment, PDA cells had altered anti- and pro-apoptotic signaling, but still responded to cabozantinib, as apoptosis only slightly decreased and viability only slightly increased suggesting a low resistance-inducing potential of cabozantinib. In parallel, c-Met expression and the pluripotency transcription factor SOX2 were downregulated, which might counteract development of full therapy resistance in long-term treated subclones. In singletreatment studies, cabozantinib increased efficacy of gemcitabine. Most importantly, cabozantinib strongly induced apoptosis and reduced viability in PDA cell lines, which are completely resistant toward gemcitabine. In primary, CSC-enriched spheroidal cultures cabozantinib downregulated CSC markers SOX2, C-Met and CD133 and induced apoptosis. These findings suggest that the clinical use of cabozantinib may be more effective than current chemotherapeutics.

Cell Death and Disease (2013) 4, e627; doi:10.1038/cddis.2013.158; published online 9 May 2013

Subject Category: Experimental Medicine

Pancreatic ductal adenocarcinoma (PDA) is one of the most aggressive malignancies usually diagnosed in an advanced state, with an extensive local invasion, early systemic dissemination and marked resistance to chemoand radiotherapy. ${ }^{1}$ The current treatment options for PDA provide a 5-year survival rate of only 5\%. Despite the low-response rate and the modest overall survival benefit and fast development of resistance, gemcitabine was adopted as the standard therapy of advanced pancreatic cancer. ${ }^{2}$ The combination of 5-fluorouracil, leucovorin, irinotecan and oxaliplatin (FOLFIRINOX), extends life by only 4 months when compared with gemcitabine. ${ }^{3}$ However, this drug combination has severe side-effects ${ }^{4}$ and neither gemcitabine nor FOLFIRINOX directly target pathways responsible for aggressive growth, early metastasis and therapy resistance of PDA.

Cancer stem cells (CSCs) are made responsible for therapy resistance and progression, as this small subpopulation within the tumor mass is thought to survive conventional cytotoxic therapy due to defense and survival mechanisms. ${ }^{5}$ CSCs are believed to possess self-renewal potential and the ability to differentiate and thereby generate the heterogeneous cell population of the originating tumor. ${ }^{6-8}$ In addition, CSCs are proposed to mediate uncontrolled growth, therapy resistance, invasion and metastasis. ${ }^{9}$ Markers for CSCs have been identified in PDA and involve a typical surface marker expression of $\mathrm{CD}_{4} 4^{+} / \mathrm{CD} 24^{+} / \mathrm{ESA}{ }^{+}$and CD133 ${ }^{+} / \mathrm{CXCR} 44^{+} .{ }^{10-12}$ The most recently detected pancreatic CSC surface marker is the mesenchymal-epithelial transition factor and membrane receptor (MET), also known as c-Met. It belongs to the group of receptor tyrosine kinases and has a key role in cell survival, growth, angiogenesis and metastasis. ${ }^{13}$ c-Met and its physiological ligand, the hepatocyte growth factor (HGF), are required for normal mammalian development and have an important role in epithelial-mesenchymal interactions during organ morphogenesis. ${ }^{13}$ The intracellular signaling cascades activated by MET include the RAS-MAPK and PI3K-AKT pathways, NF- $\kappa$ B and Wnt/GSK- $3 \beta / \beta$-Catenin signaling. ${ }^{13}$ Many carcinomas overexpress MET and the surrounding stroma overexpresses HGF. c-Met is also overexpressed in pancreatic cancer and FACS-sorted $\mathrm{c}^{-\mathrm{Met}^{+}}$primary pancreatic cancer cells exhibit higher tumor-take in mice than the c-Met population. ${ }^{14}$ These findings have led to the conclusion that c-Met is a pancreatic CSC marker.

\footnotetext{
${ }^{1}$ Department of Experimental Medicine, Molecular OncoSurgery Group, University of Heidelberg and German Cancer Research Center, Heidelberg, Germany; ${ }^{2}$ Department of General Surgery, Institute for Immunology, University of Heidelberg, Heidelberg, Germany and ${ }^{3}$ Department of Molecular Immunodiagnostics, Institute for Immunology, University of Heidelberg, Heidelberg, Germany

${ }^{*}$ Corresponding author: I Herr, Department of Experimental Medicine, Molecular OncoSurgery Group, University of Heidelberg and German Cancer Research Center DKFZ-G403, Im Neuenheimer Feld 365, Heidelberg 69120 Germany. Tel: +49 622156 5147; Fax: +49 622156 6119; E-mail: i.herr@dkfz.de

Keywords: cancer stem cells; pancreatic cancer; XL184

Abbreviations: XL184, Cabozantinib; CSC, Cancer stem cell; PDA, Pancreatic ductal adenocarcinoma

Received 23.3.13; revised 09.4.13; accepted 10.4.13; Edited by A Stephanou
} 


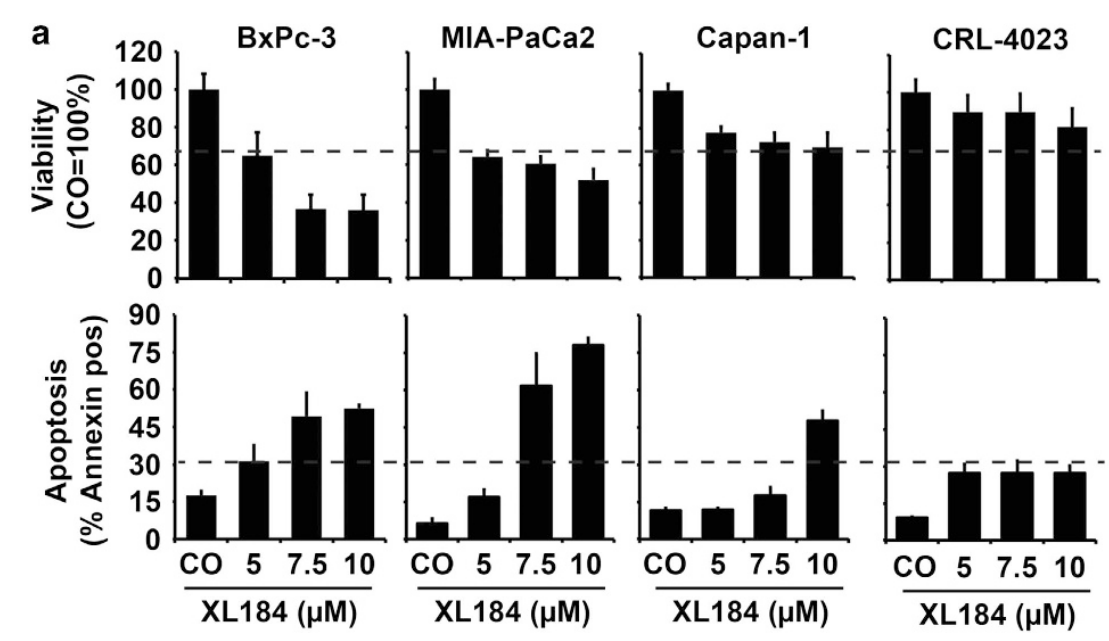

b
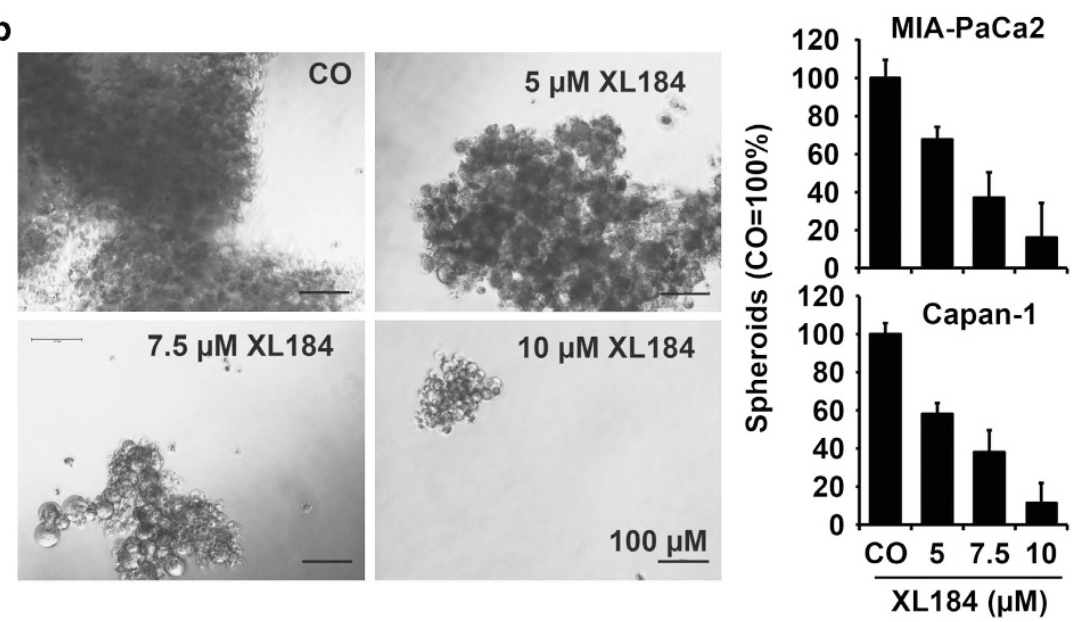

Figure 1 Cabozantinib eliminates PDA cells but is less toxic to non-malignant ductal cells. (a) Cells were left untreated or were treated with $5,7.5$ or $10 \mu \mathrm{m}$ cabozantinib (XL184). Seventy-two hours later viability was detected by MTT-assay and apoptosis by staining with annexinV-FITC followed by FACS-analysis. (b) MIA-PaCa2 and Capan-1 cells were cultured in ultra-low attachment plates for spheroid formation. Three days later cells were treated as described above and as indicated. Ten days after treatment spheroids were evaluated morphologically by microscopy and the percentage of spheroids in each group were determined by counting viable spheroidal cells. The number of spheroids in the control was set to $100 \%$. Representative pictures of MIA-PaCa2 spheroids are shown

Currently, the therapeutic potential of the c-Met inhibitor cabozantinib (XL184), developed by Exelixis, is intensively investigated. Cabozantinib is a potent dual inhibitor of c-Met and VEGFR-2 signaling. ${ }^{12,15}$ Pre-clinical studies were performed in spontaneous pancreatic islet tumors in RIP-Tag2 transgenic mice, where cabozantinib led to rapid, widespread and progressive regression of tumor vasculature, extensive hypoxia and apoptosis of tumor cells and decreased tumor aggressiveness. ${ }^{15}$ In human pancreatic tumors growing orthotopically in NOD SCID mice, cabozantinib slowed tumor growth and reduced the population of CSCs when given alone or in combination with gemcitabine. ${ }^{12}$ Administration of XL184 for 2 weeks after cardiac injection of pancreatic cancer cells prevented the development of metastases. ${ }^{12}$ The clinical efficacy of cabozantinib in several progressed tumor entities is under investigation in randomized phase II studies, including patients with metastasizing pancreatic cancer. ${ }^{16}$ On 29 November, 2012, cabozantinib (Cometriq) was approved by the FDA for the treatment of patients with progressive medullary thyroid carcinoma. ${ }^{17}$ Despite ongoing studies in patients, it is still completely unknown if cancer cells may develop extrinsic therapy resistance in response to cabozantinib treatment, as known for current cytotoxic therapies, and if cabozantinib might be able to overcome resistance to current cytotoxic therapy.

In the present work, we demonstrate that repeated cycles with cabozantinib induced a low-level of therapy resistance, which was, however, much less pronounced than resistance induced by gemcitabine. Cabozantinib altered the expression of apoptosis molecules and shifted the balance to antiapoptotic signaling. In parallel, cabozantinib inhibited SOX2, c-Met and CD133 expression and the self-renewal potential. Most importantly, cabozantinib increased efficacy of gemcitabine even in high-gemcitabine-resistant PDA cells and in patient-derived primary spheroidal cultures enriched in CSC markers.

\section{Results}

Cabozantinib effectively eliminates PDA cells but is less toxic to non-malignant ductal cells. To evaluate the therapeutic effect of cabozantinib we used three established 
human PDA cell lines. According to the degree of differentiation of the primary tumor, mutations in KRas and/or p53, in vitro morphology, colony- and spheroid-forming capacity, ALDH activity, tumorigenicity in mice, histology of xenografts and expression of E-cadherin and Vimentin we classified MIA-PaCa2 and Capan-1 as CSC ${ }^{\text {high }}$ and $\mathrm{BxPC}-3$ as $\mathrm{CSC}^{\text {low }}$ (Supplementary Table S1). The cell line BxPc-3-GEM, is a gemcitabine-resistant subclone of parental BxPc-3 cells and classified as CSC ${ }^{\text {median }}$. FACS-analysis ensured that c-Met is expressed in all cells used (Supplementary Figure 1). Treatment with $5,7.5$ or $10 \mu \mathrm{m}$ cabozantinib resulted in downregulation of viability and upregulation of apoptosis

a

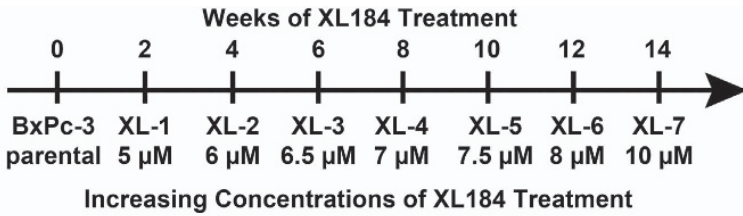

b
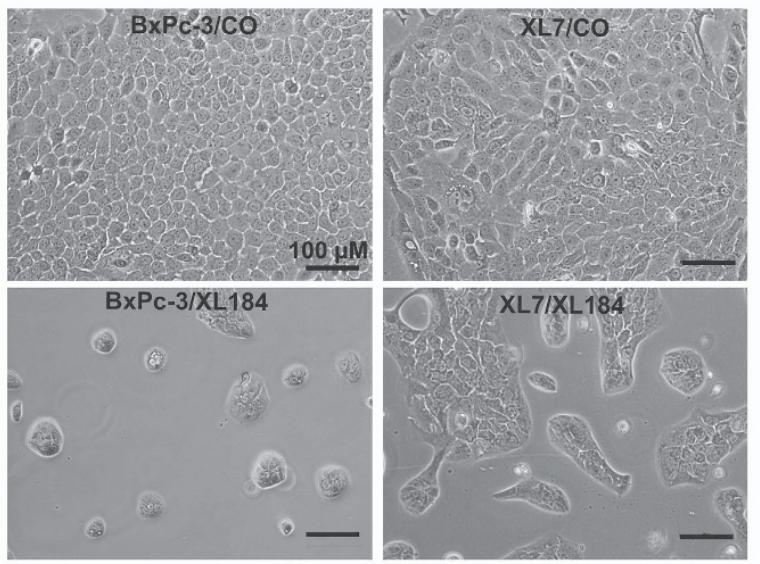

C
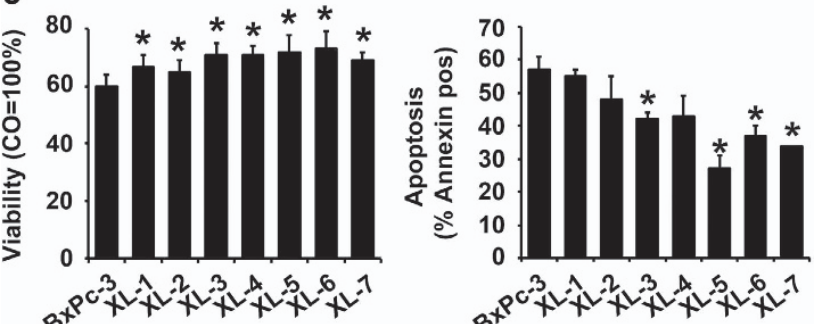

$3 \times 2+3+5.61$

$88^{c^{3}}+x^{2}+2^{2}+x^{2}+2^{2}+x^{2}+x^{2}+2^{2}$

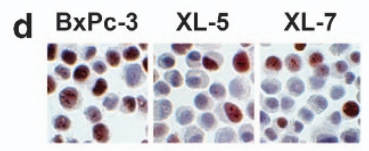

e $B x P c-3 \quad X L-5 \quad x L-7$
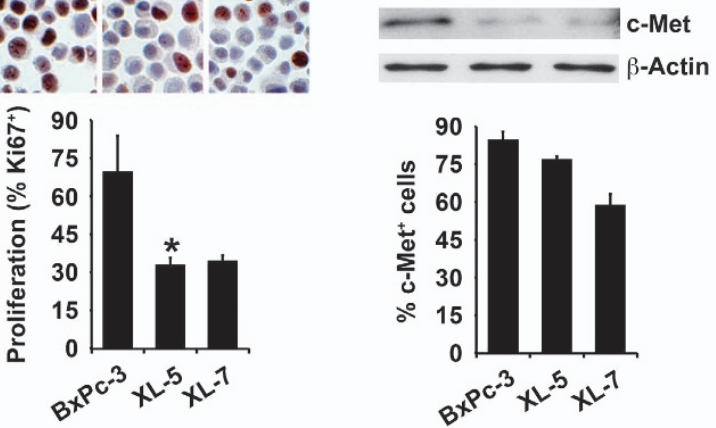

within $72 \mathrm{~h}$, as measured by MTT-assay and annexinstaining followed by FACS-analysis (Figure 1a). Higher concentrations of 12.5 and $15 \mu \mathrm{m}$ cabozantinib did not further reduce viability (data not shown). Downregulation occurred in a dose-dependent manner with most pronounced effects in $\mathrm{BxPc}-3$ cells, followed by MIA-PaCa2 and Capan-1. In contrast, cabozantinib was less toxic in non-malignant CRL-4023 cells, as it influenced viability and apoptosis only marginally. To detect the influence of cabozantinib on self-renewal potential, MIA-Paca2 and Capan-1 cells were cultivated in ultra-low attachment plates for spheroid formation. BxPc-3 cells were not used because this less aggressive cell line does not grow anchorage-independent. After the formation of spheroids, cells were treated with increasing concentrations of cabozantinib. After 10 days the number of spheroids in each group was determined microscopically (Figure 1b). Cabozantinib destroyed spheroid formation in a dose-dependent manner, which was almost complete at the highest concentration of $10 \mu \mathrm{m}$ cabozantinib. Together, all three malignant pancreatic cancer cells are sensitive to cabozantinib treatment, while non-malignant cells mostly resist.

Long-term treatment with cabozantinib induces minor resistance. A major problem in pancreatic cancer is a highintrinsic resistance, in addition to extrinsic resistance, which develops with repeated cycles of standard chemotherapy, ${ }^{1,2}$ resulting in a total resistance to for example, gemcitabine. ${ }^{18}$ Although more differentiated cells die upon chemotherapy, the small population of CSCs is resistant and survives and is therefore enriched with each new treatment cycle. ${ }^{19}$ Thus, new chemotherapeutic agents should target the CSC population. For evaluation of induction of resistance toward cabozantinib, we mimicked the clinical treatment: BxPc-3 cells were treated with $5 \mu \mathrm{m}$ cabozantinib, followed by 2 weeks recovery for surviving cells resulting in the subclone

Figure 2 Long-term treatment with cabozantinib induces minor resistance. (a) Scheme of long-term treatment of BxPc-3 cells with cabozantinib and establishment of subclones XL-1 to XL-7. BxPc-3 cells were treated seven times with increasing concentrations of cabozantinib $(5-10 \mu \mathrm{m})$ over 14 weeks. After each treatment, cells were allowed to recover for 2 weeks before the next treatment with increased concentration was applied. Two weeks after each treatment respective subclones were obtained that were either left untreated for examination of resistance or used for the next treatment round. (b) Parental BxPc-3 cells or the derived subclone XL-7 were treated with $10 \mu \mathrm{m}$ cabozantinib. Seventy-two hours later cells were analyzed with a Nikon Eclipse TS100 microscope and $\times 100$ magnification and photographed. (c) Parental BxPc-3 cells or the derived subclones $\mathrm{XL}-1$ to XL-7 were treated with $10 \mu \mathrm{m}$ cabozantinib. Seventy-two hours later viability was analyzed by MTT-assay and controls for each cell line were set to $100 \%$. Apoptosis was analyzed by staining with annexinV-FITC and FACS-analysis. The percentage of specific apoptosis was calculated by the formula: $100 \times$ (((apoptosis of treated cells)-(spontaneous apoptosis of control cells))/(100-spontaneous apoptosis of control)) ( ${ }^{*} p<0.05$ compared with control; calculated using a twosided Student's t test). (d) BxPc-3, XL-5 and XL-7 cells were treated with $10 \mu \mathrm{m}$ cabozantinib. Seventy-two hours later cells were cytospinned to glass slides and expression of the proliferation marker Ki67 was detected by immunohistochemistry and photographs are shown. The number of positive cells was quantified in 10 vision fields under $\times 400$ magnification and the means \pm S.D. are shown in a diagram. $\left({ }^{*} p<0.05\right.$ compared with control) (e) Likewise, $c-M e t$ expression was analyzed by western blot analysis or by evaluation of positively-stained cells in immunohistochemistry as described in point (d) 

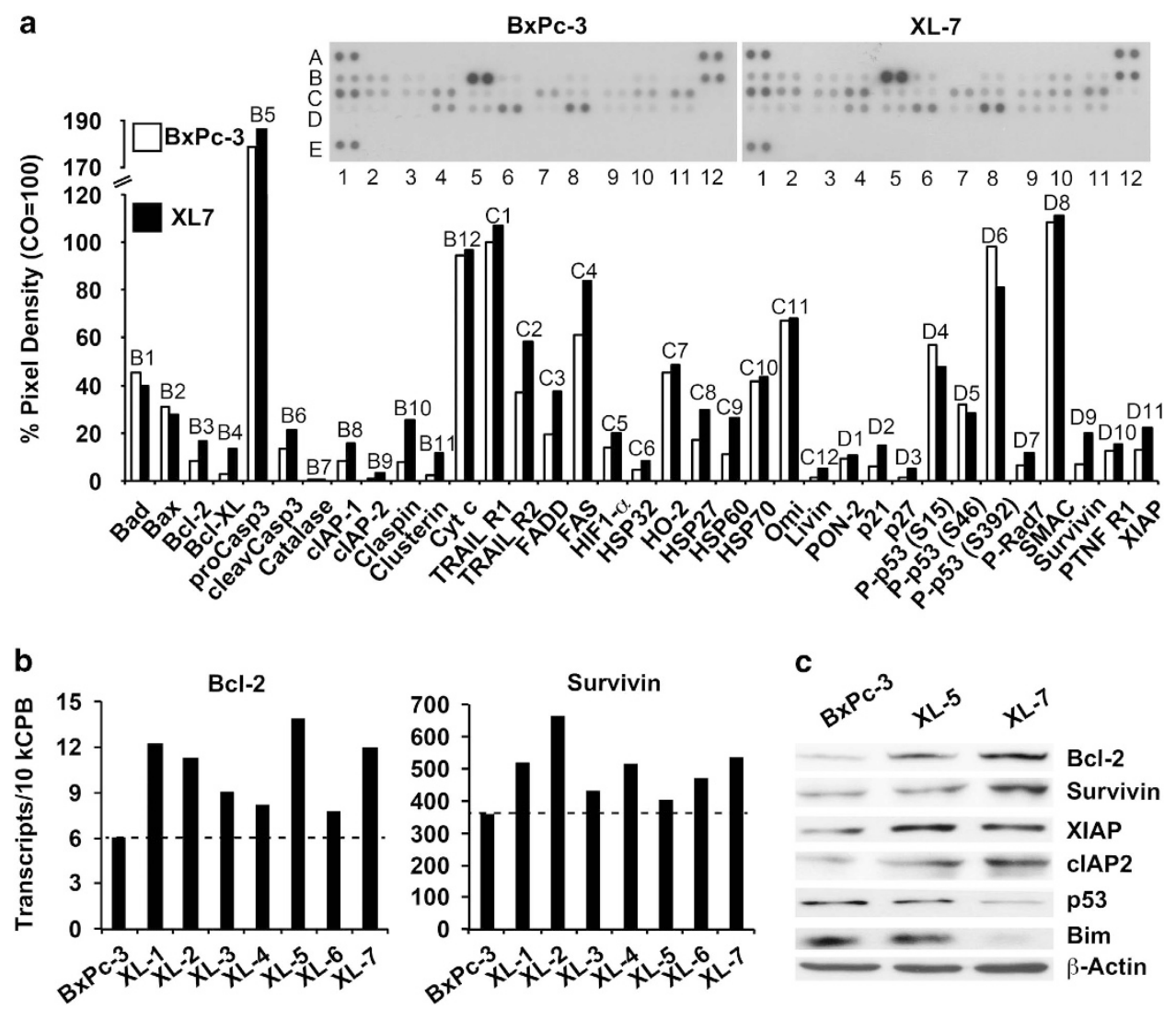

Figure 3 Long-term treatment with cabozantinib changes the level of anti- and pro-apoptotic proteins. (a) Proteins were isolated from parental BxPc-3 cells and the derived subclone XL-7. Binding of proteins to antibodies spotted in duplicate to the membrane of a Human Apoptosis Array was detected using biotinylated secondary antibodies, streptavidin-HRP and chemiluminescence. The pixel density was quantified using ImageJ software and normalized to the mean pixel intensity of the reference spots A1, A12 and E1 on the membrane. Spot D12 is the PBS-negative control. (b) mRNA from parental BxPc-3 cells and derived subclones XL-1 to XL-7 was harvested and expression of Bcl-2 and Survivin were examined by qRT-PCR. (c) Likewise, expression of anti-apoptotic (Bcl-2, Survivin, XIAP, clAP2) and pro-apoptotic (p53, Bim) proteins were examined by western blot analysis

$\mathrm{XL}-1$ (Figure 2a). XL-1 were treated with $6 \mu \mathrm{m}$ cabozantinib followed by 2 weeks recovery for surviving cells resulting in the subclone XL-2. These rounds of treatment were repeated seven times with consecutively enhanced concentrations of cabozantinib resulting in the subclone XL-7 and treatment with $10 \mu \mathrm{m}$ cabozantinib after 14 weeks. Treatment of parental BxPc-3 or the selected subclones with $10 \mu \mathrm{m}$ cabozantinib induced apoptosis and reduced viability in all cell lines as detected by microscopic evaluation of cells, MTT-assay and annexin-fluoresceinisothiocyanate (FITC)staining of exposed phosphatidylserine followed by FACSanalysis (Figures $2 \mathrm{~b}$ and $\mathrm{c}$ ). However, as higher the treatment round as lower was the reduction of viability and the induction of apoptosis, although in XL-7 induction of apoptosis was still $30 \%$ and reduction of viability $70 \%$ versus $57 \%$ and $60 \%$ in parental BxPc-3 cells, respectively. Modest downregulation of cabozantinib sensitivity was associated with downregulation of the proliferation marker $\mathrm{Ki} 67$ as determined by immunohistochemistry of untreated parental BxPc-3 cells and derived XL-5 and XL-7 subclones cytospinned to glass slides (Figure 2d). Quite similarly, c-Met expression was downregulated in untreated XL-5 and XL-7 subclones compared with parental BxPc-3 cells and as measured by immunohistochemistry and western blot analysis (Figure 2e). This was associated with enhanced expression of pro-apoptotic markers (e.g. cleaved Caspase3, TRAIL R2, FAS) while some anti-apoptotic markers were upregulated (e.g. Bcl-2, IAP-1, Survivin and XIAP) as measured by protein antibody array (Figure $3 a$ ). Array results were randomly confirmed by qRT-PCR analysis of Bcl-2 and Survivin mRNA expression (Figure $3 b$ ) and by western blot analysis of Bcl-2, p53, clAP2, Survivin and XIAP expression (Figure $3 \mathrm{C}$ ). In addition, the pro-apoptotic proteins Bim and p53 were downregulated in XL-7 subclones. These results suggest that long-term treatment with cabozantinib leads to a change in expression of pro- and anti-apoptotic molecules, which is most likely responsible for the observed slight increase in resistance to long-term repeated treatment with cabozantinib (compare Figures $2 b-d$ ).

Long-term treatment with cabozantinib reduces stem cell-associated signaling. To elucidate the effect of longterm cabozantinib treatment to CSC-associated signaling we detected levels of proteins involved in pluripotency and progression of cancer by an antibody array (markers of this array are summarized in Supplementary Table 2). By examination of protein extracts from parental BxPc-3 and the derived $\mathrm{XL}-5$ and $\mathrm{XL}-7$ cells we found strong 

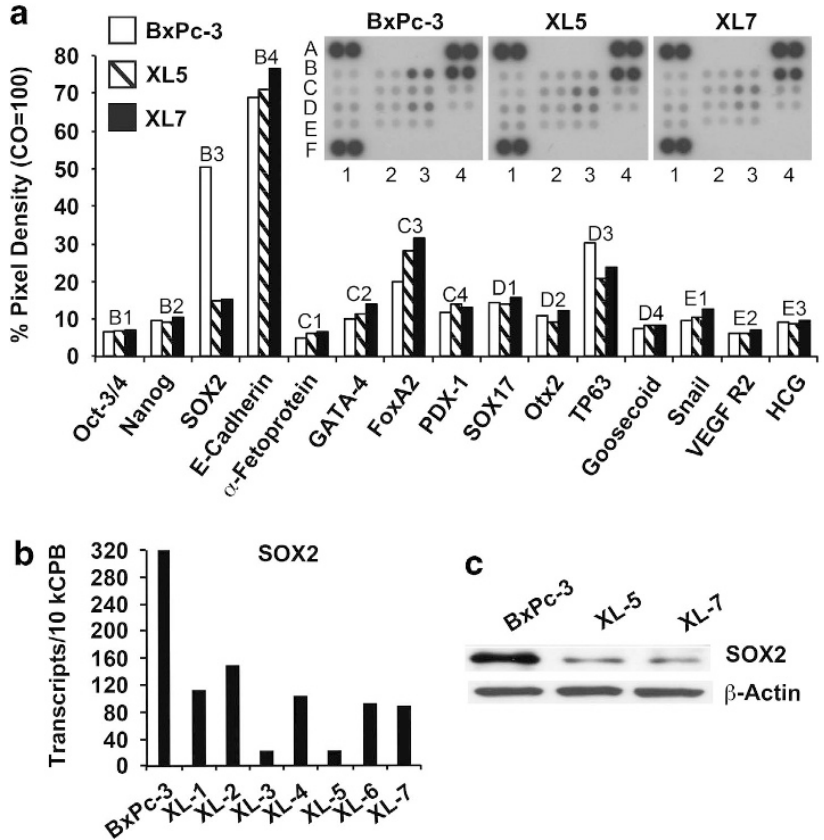

Figure 4 Long-term treatment with cabozantinib downregulates SOX2. (a) Proteins were isolated from parental $\mathrm{BxPc}-3$ cells and the derived subclones XL-5 and XL-7. Binding of proteins to antibodies spotted in duplicate to the membrane of a Human Pluripotent Stem Cell Array was detected as described in Figure 3a. Reference spots are located at the coordinates A1, A4 and F1. Spot E4 is the PBSnegative control. (b) mRNA from parental BxPc-3 cells and derived subclones XL-1 to XL-7 were harvested and expression of SOX2 was examined by qRT-PCR. (c) Likewise, protein expression of SOX2 was examined by western blot analysis

downregulation of SOX2 in long-term cabozantinib treated subclones (Figure 4a). Downregulation of SOX2 was confirmed by qRT-PCR and western blot analysis (Figures 4b and c). Likewise, forkhead box protein A2 (FoxA2) levels were upregulated in XL-5 and XL-7 subclones. Similarly, protein levels of p63, a member of the p53 family, ${ }^{20}$ were downregulated, according to the results shown in Figure 3a, where downregulation of p53 proteins phosphorylated at serine 15, 46 or 392 was found. Together, downregulation of the reprogramming transcription factor SOX2 suggests diminished self-renewal potential, ${ }^{21,22}$ while upregulation of FOXA2 is associated with reduced epithelial-mesenchymal transition ${ }^{23}$ and loss of $p 63$ may increase tumorigenesis. ${ }^{20}$

Cabozantinib inhibits self-renewal potential and increases gemcitabine efficacy. For comparison of the therapeutic efficacy of cabozantinib and gemcitabine, BxPc-3, MIA-PaCa2 and Capan-1 cells were treated with gemcitabine and cabozantinib alone or combined. Seventytwo hours later diminished viability and enhanced apoptosis occurred in cells upon single treatment while combination treatment was more effective (Figure 5a). This result was confirmed by colony-forming assays in BxPc- 3 cells and by spheroid assays in MIA-PaCa2 and Capan-1 cells, where each single treatment reduced self-renewal potential, which was, however, only complete after combination therapy (Figures $5 \mathrm{~b}$ and c). Next we examined whether cabozantinib might be able to overcome gemcitabine resistance. BxPc-3GEM cells totally resistant to gemcitabine and treatment with gemcitabine for $72 \mathrm{~h}$ did not reduce the number of cells and viability or induced cell death and apoptosis (Figure 6a). In contrast, cabozantinib strongly reduced cell number and viability along with inducing cell death and apoptosis. These effects were even more pronounced upon combination of cabozantinib with gemcitabine. In addition, cabozantinib led to strong downregulation of $\mathrm{c}$-Met and its phosphorylated form along with downregulation of SOX2, cIAP2 and Survivin (Figure 6c), whereas gemcitabine had only minor effects to expression of these proteins. However, combination of gemcitabine and cabozantinib resulted in nearly total downregulation. Correspondingly, cabozantinib but not gemcitabine strongly induced cleavage of caspase-3 and reduced proliferation with no significant further increase upon combination treatment (Figure $6 \mathrm{~d}$ ). These results reveal that cabozantinib overcomes chemotherapy resistance of pancreatic cancer cells by induction of apoptosis signaling and downregulation of c-Met- and SOX2 signaling.

\section{Cabozantinib reduces stem cell markers and induces} apoptosis in patient-derived primary spheroidal cultures. To further highlight these findings in a patientrelated model, we transplanted freshly resected human PDA tissue (Figure 7a) subcutaneously to mice followed by serial subtransplantation. With increasing passage number starting at passage 3 the xenograft tumors started to grow faster while the typical duct-like structures and the pronounced tumor stroma typical for PDA were preserved (data not shown). Tumor cells were isolated from xenografts of two different tumors (T30, T18) and cultured in vitro as anchorage-independent spheroidal colonies. Spheres were cultured with gemcitabine, cabozantinib or both together, followed by preparation of cytospins $72 \mathrm{~h}$ later. In untreated spheroids, immunohistochemistry revealed the presence of $70 \% \mathrm{c}^{-M e t}{ }^{+}$cells and of $80 \% \mathrm{CD} 133^{+}$cells suggesting strong enrichment of CSCs in these cultures (Figure 7b). Although gemcitabine was minor effective, cabozantinib alone or combined with gemcitabine strongly reduced the expression of CD133 and c-Met. This was associated with downregulation of SOX2 and upregulation of cells undergoing apoptosis, as concluded from an enhanced number of spheroids positive for the cleaved form of caspase-3 protein. These data indicate that cabozantinib eliminates features of CSCs even in patient-derived, primary CSC-enriched spheroidal cultures.

\section{Discussion}

In the present study, we show that the c-Met inhibitor cabozantinib targets stem cell-features in pancreatic cancer and thereby induces apoptosis and increases the efficacy of gemcitabine. This effect occurs even in PDA cells, which are completely resistant to gemcitabine. Most importantly, longtime repeated treatment with cabozantinib did not induce pronounced apoptosis resistance as known from long-term gemcitabine treatment. Although we observed a shift to antiapoptotic signaling in response to 14 weeks of cabozantinib treatment, downregulation of CSC markers SOX2, CD133 and c-Met occurred in parallel and may prevent development of prounounced resistance. 
Studies in human cells and tissues have shown that c-Met is overexpressed in a variety of human cancers, including pancreatic cancer. ${ }^{24-26} \mathrm{~A}$ study by Li et al. ${ }^{12}$ identified c-Met as a new marker for pancreatic CSCs and defined the c-Metpositive population as the tumorigenic one. The same group found that cabozantinib treatment of mice with xenotransplanted human PDA tissue led to reduction of tumor growth and a block of metastasis. While cabozantinib or gemcitabine alone were capable of inhibiting tumor growth, combined

a
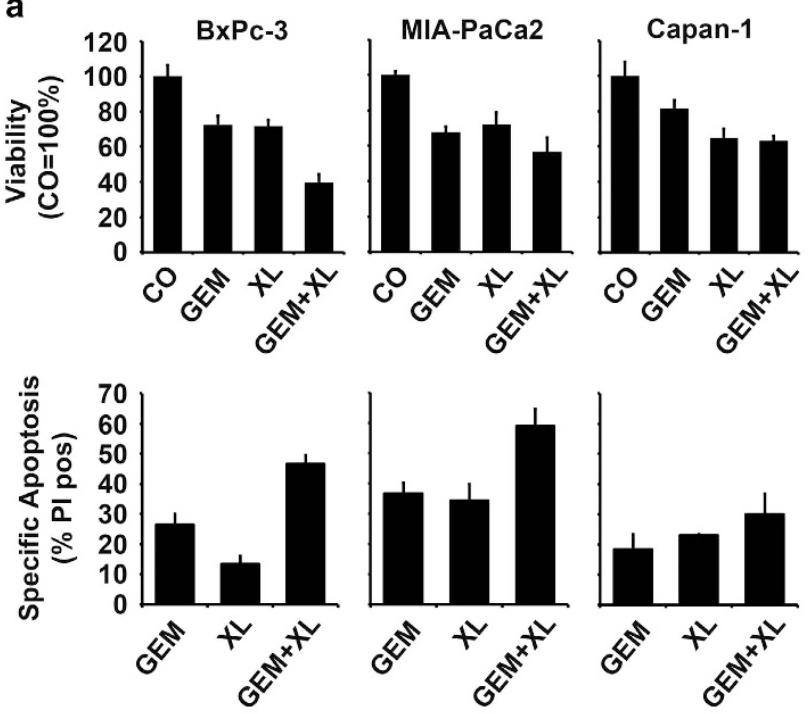

b
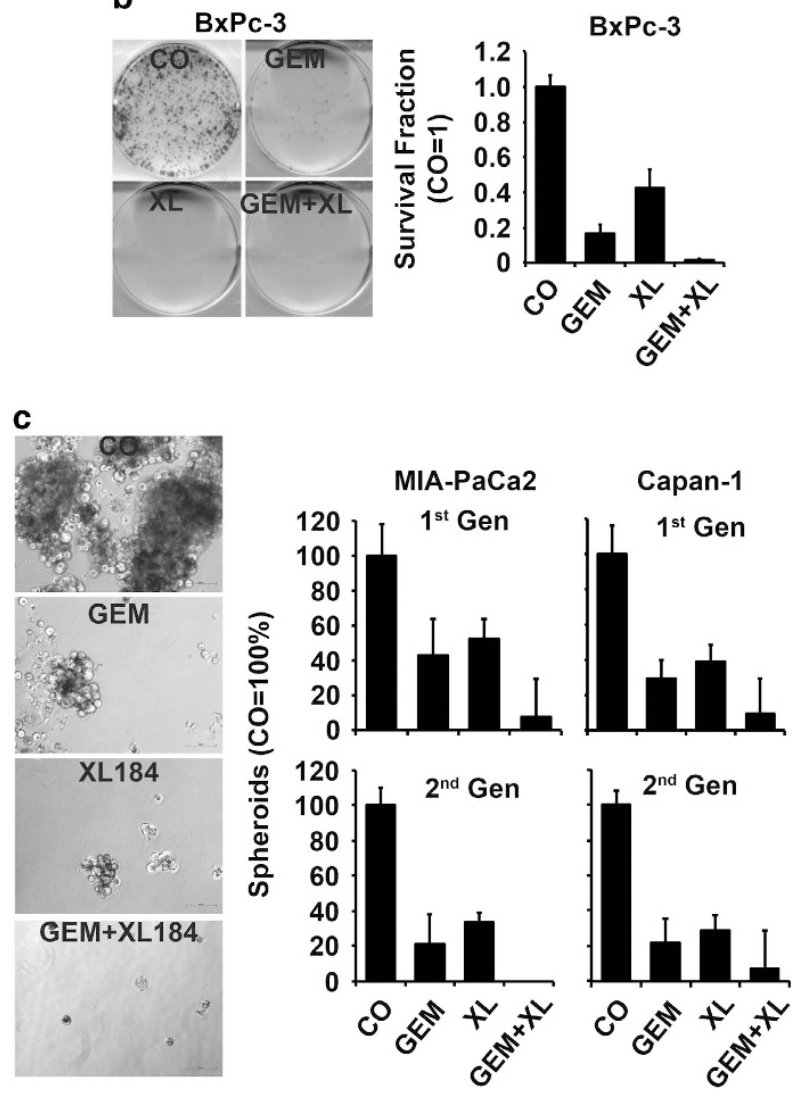

treatment completely blocked tumor growth in mice, even up to 32 days after cessation of treatment. ${ }^{12}$ These data are in line with our in vitro results showing that cabozantinib and gemcitabine inhibit viability and enhance apoptosis in gemcitabine-sensitive cells. In addition, we demonstrate that selfrenewal potential is targeted due to inhibition of colony- and spheroid-formation with most pronounced effects in combination therapy. Most importantly, we found that cabozantinib is even effective in PDA cells, which are totally resistant toward gemcitabine. In contrast, cabozantinib cytotoxity was negligible in non-malignant pancreatic ductal cells, which suggests low-side effects. Clinical side effects have been already evaluated in a study where cabozantinib (Cometriq) was applied to 330 patients with medullary thyroid carcinoma. ${ }^{27}$ Adverse side effects of cabozantinib treatment included diarrhea, stomatitis, palmar-plantar erythrodysesthesia syndrome, decreased weight, decreased appetite, nausea, fatigue, oral pain, hair color changes, dysgeusia and hypertension. ${ }^{27}$ Despite these side-effects the outcome was promising as tumor progression was inhibited and in some patients even the tumor size was reduced.

In contrast to the observed development of complete extrinsic resistance toward gemcitabine, repeated treatment with increasing concentrations of cabozantinib over 14 weeks induced minor resistance. This is concluded in part from our qRT-PCR experiment for that we have collected cell pellets after each of the seven treatment rounds to isolate RNA and to perform the qRT-PCR at the end of the experiment. Our results clearly show that $\mathrm{Bcl}-2$ and Survivin is upregulated and the expression of SOX2 is downregulated in all treatment rounds compared with untreated control cells. Although the variability in the qRT-PCR experiment is high as we could not repeat the experiment due to limited sample availability, we confirmed the data by protein array and western blot analysis. These experiments together demonstrate that cabozantinib leads to upregulation of anti-apoptototic proteins $\mathrm{Bcl}-2$, Survivin, XIAP and clAP, and downregulation of the pro-apoptotic proteins p53 and Bim. This tendency to anti-apoptotic signaling was, however, counteracted by

Figure 5 Cabozantinib inhibits self-renewal potential and increases gemcitabine efficacy. (a) Parental BxPc-3 cells were left untreated or were treated with $10 \mu \mathrm{m}$ cabozantinib (XL), $50 \mathrm{~nm}$ gemcitabine (GEM) or both together (GEM $+\mathrm{XL}$ ). Seventy-two hours later viability and apoptosis were analyzed as described in Figure $2 \mathrm{~b}$, except of that apoptosis was detected by staining with propidium iodide (PI) instead of annexinV-FITC. (b) Parental BxPc-3 cells were treated as described in point (a). Seventy-two hours later cells were replated to six-well plates $(2000 \mathrm{cells} /$ well). Cells were grown without a medium change for 2 weeks, followed by evaluation of fixed and coomassie-stained colonies consisting of at least 50 cells. The plating efficiency was calculated using the following formula: number of colonies/number of seeded cells and presented as Survival Fraction. (c) MIA$\mathrm{PaCa} 2$ and Capan-1 cells were seeded at clonal density in low-adhesion plates for spheroid formation. Three days later cells were treated as described above in point (a). Seven days after treatment, the percentage of viable spheroids in each group were determined by counting viable spheroidal growing cells of 1st-generation (1st Gen). The number of spheroids in the control was set to $100 \%$. Thereafter, the 1 stgeneration spheroids were dissociated to single cells, and equal numbers of live cells were seeded at clonal density in low-adhesion plates resulting in 2ndgeneration spheroids (2nd Gen), which were treated 3 days after seeding. The 2ndgeneration spheroids were grown until day 7 after treatment and photographed at $\times 100$ magnification or quantified as described above 
a
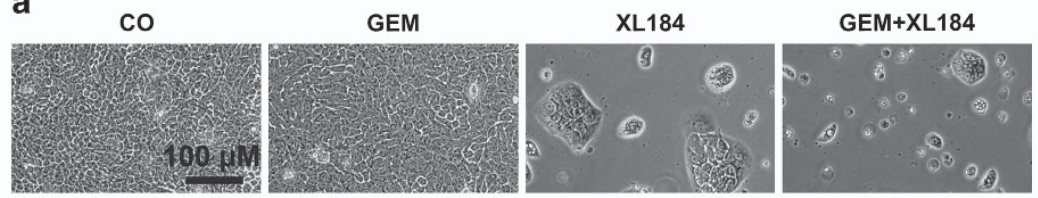

b
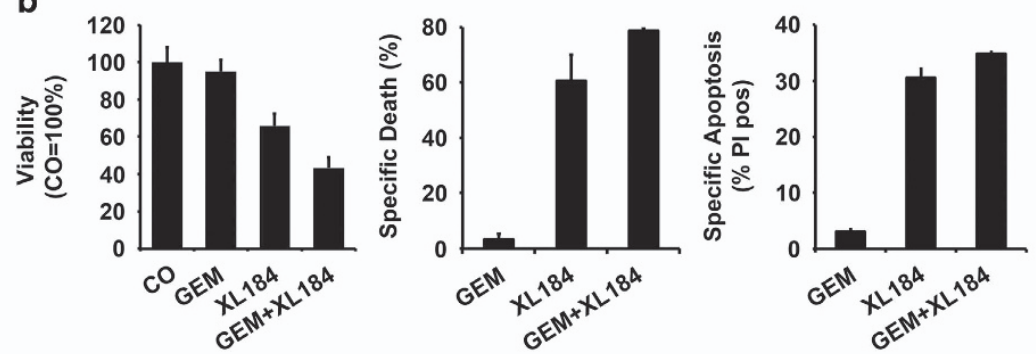

C

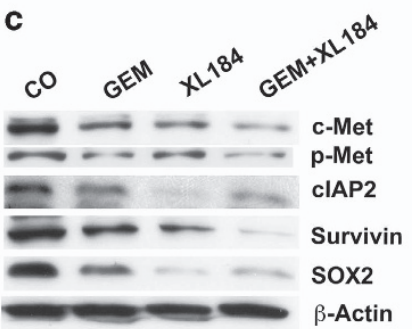

Figure 6 Cabozantinib overcomes gemcitabine resistance. (a) BxPc-3-GEM cells, which are a BxPc-3-derived and gemcitabine-resistant subclone, were left untreated or were treated with $10 \mu \mathrm{m}$ cabozantinib (XL), $50 \mathrm{~nm}$ gemcitabine (GEM) or both together (GEM $+\mathrm{XL}$ ). Seventy-two hours later morphology was photographed at $\times 100$ magnification. (b) At the same time point after treatment viability and apoptosis were analyzed as described in Figure $5 \mathrm{a}$, and cell death was evaluated by examining granularity of cells by FACS-analysis. Specific death was calculated according to the formula given in Figure 2c. (c) Seventy-two hours after treatment, the expression of c-Met, phosphorylated Met (p-Met), cIAP2, Survivin and SOX2 were analyzed by western blot. (d) Likewise, seventy-two hours after treatment, cells were cytospinned to glass slides and the presence of cells positive for cleaved caspase-3 or Ki67 were analyzed by immunohistochemistry to detect apoptosis and viability, respectively. Photographs of positive cells (red and dark brown) are shown. The number of positive cells was quantified in 10 vision fields under $\times 400$ magnification and the means \pm S.D. are shown

cabozantinib-mediated strong downregulation of the reprogramming transcription factor SOX2, involved in embryogenesis and self-renewal of stem cells. ${ }^{28} \mathrm{~A}$ correlation between c-Met and SOX2 is suspected ${ }^{14}$ and may explain why we observed diminished self-renewal potential after inhibition of c-Met signaling or why inhibition of metastasis was observed in a mouse model. ${ }^{12}$ Mechanistically, c-Met activation is reported to induce the expression of the stem cell reprogramming transcription factors SOX2, c-Myc, KIf4, Oct4 and Nanog and thereby counteracts the differentiation of glioblastoma neurospheres. ${ }^{14}$ Most likely, c-Met-induced stem cell reprogramming is also involved in generation of pancreatic CSCs capable of self-renewal and cabozantinib interrupts this process.

In addition, cabozantinib downregulated proliferation as detected by Ki67 staining. Downregulated proliferation may have contributed to slight but not pronounced induction of apoptosis resistance in response to continuous treatment with cabozantinib as general agreement exists that cytotoxicity of anticancer agents is proliferation-dependent. ${ }^{29}$ Simultaneously, we observed downregulation of expression of c-Met and CD133 in response to cabozantinib. In case of c-Met downregulation, we think that this is rather due to inhibited expression of $\mathrm{c}$-Met rather than to apoptosis of
c-Met-positive cells. The reason for this assumption is that we extracted proteins only of alive cells and excluded dead cells for western blot and antibody protein array analysis. Downregulation of c-Met may be due to a positive feedback loop, as autocrine/paracrine mechanisms of c-Met hyperactivation including receptor and/or HGF overexpression in multiple solid malignancies has been shown. ${ }^{26}$ The mechanism, by which cabozantinib leads to downregulation of CD133 expression is less clear, but may be linked to downregulation of reprogramming transcription factor SOX2. ${ }^{28}$

In our study, we did not examine whether co-treatment of pancreatic cancer cells with cabozantinib and gemcitabine may prevent development of gemcitabine resistance. However, we found inhibition of CSC characteristics by cabozantinib without development of pronounced resistance to cabozantinib itself. Therefore, our data strongly point to the direction that co-treatment may prevent development of gemcitabine resistance due to cabozantinib-mediated prevention of enrichment of CSCs during repeated gemcitabine treatment.

Undoubtedly, further studies will be needed to determine the precise mechanisms, by which cabozantinib potently eliminates malignant pancreatic cancer cells while sparing more differentiated cells. Regardless, our data provide strong 
a

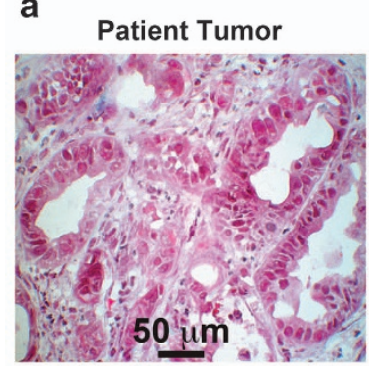

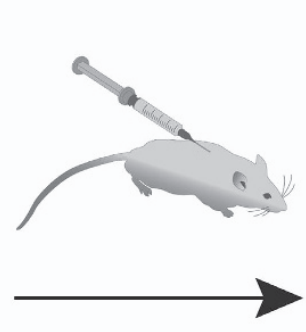

Primary Spheroids

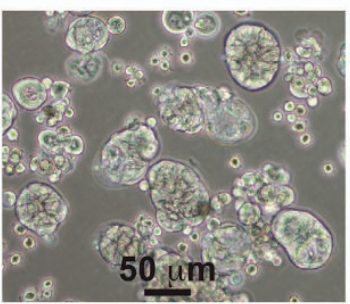

b

$\square$ CO $\square$ GEM

XL

GEM+XL

Spheroidal Cells T30

\section{Spheroidal Cells T18}
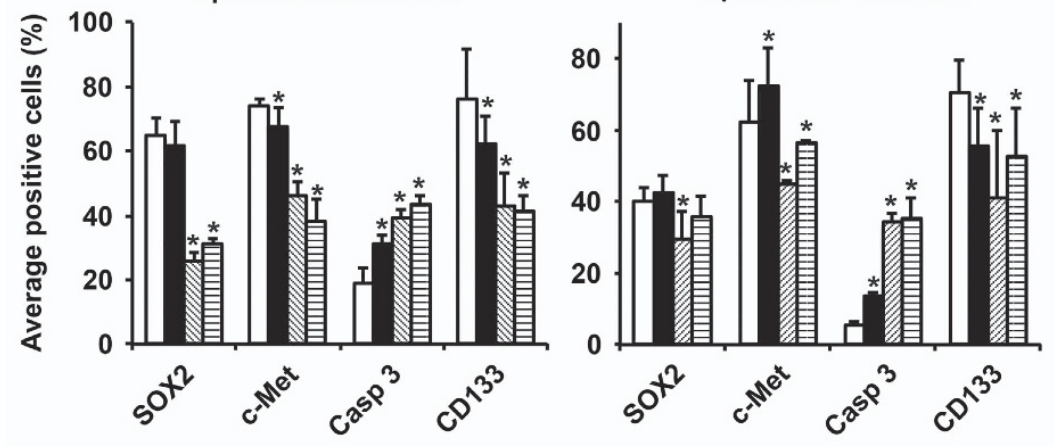

Sox2

c-Met
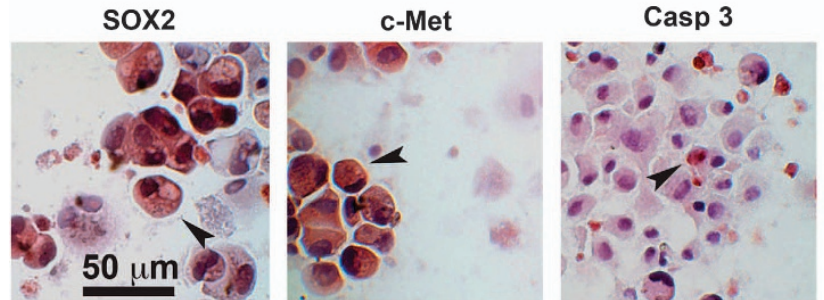

CD133

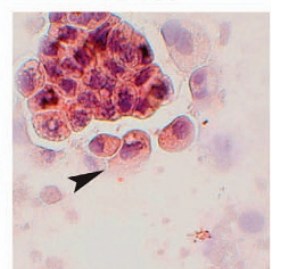

Figure 7 Cabozantinib prevents growth of patient-selected and CSC-enriched spheroids. (a) Tumor mush isolated from surgical non-diagnostic specimens of patients with PDA was transplanted subcutaneous to the flanks of immunodeficient 6-week-old NMRI (nu/nu) female mice followed by subtransplantation until a stable growing xenograft line developed after passage 3. Morphology of the primary patient tumors was detected by Trichrome staining of frozen tissue followed by microscopy. Anchorage-independent spheroidal cultures were established from stably-growing xenograft lines and were left either untreated or were treated with $10 \mu \mathrm{m}$ cabozantinib, $50 \mathrm{~nm}$ gemcitabine or both together for $72 \mathrm{~h}$. (b) Treated spheroidal cultures were cytospinned to glass slides and CSC marker expression (c-Met, CD133, SOX2) and activity of caspase-3 (Casp3) were examined by immunohistochemistry. The number of positive cells was quantified in 10 vision fields under $\times 400$ magnification and the means \pm S.D. are shown $\left({ }^{*} p<0.05\right.$ compared with control). Arrowheads indicate positive cells

evidence that cabozantinib-mediated downregulation of c-Met expression followed by downregulation of SOX2 expression may be key factors in overcoming resistance toward gemcitabine and in avoiding development of prounounced extrinsic resistance after repeated therapy cycles of cabozantinib.

\section{Materials and Methods}

Cell lines. Human established PDA cells BxPc-3, MIA-PaCa2 and Capan-1 and human hTERT-HPNE immortalized pancreatic duct cells CRL-4023 were from the American Type Culture Collection (Manassas, VA, USA). Gemcitabine-resistant BxPc-3 cells (BxPc-3-GEM) were selected by continuous treatment of BxPc-3 cells during several months in increasing concentrations of gemcitabine up to $100 \mathrm{~nm} .{ }^{18,30}$ Cells were cultured in DMEM (PAA, Pasching, Austria) supplemented with $10 \%$ heat-inactivated FCS (Sigma, Deisenhoffen, Germany) and $25 \mathrm{mmol} / \mathrm{l}$ HEPES (PAA). Cells were authenticated throughout the culture by the typical morphology. To maintain authenticity of the cell lines, frozen stocks were prepared from initial stocks, and every 3 months a new frozen stock was used for the experiments. Mycoplasma-negative cultures were ensured by monthly testing.

Selection of CSC-enriched spheroidal cells from patient tumors by subtransplantation. A surgical non-diagnostic specimen was mechanically minced and $2 \times 10^{7}$ cells in matrigel were transplanted into the flanks of 6-week-old NMRI (nu/nu) female mice. After development of a tumor, the xenograft was resected, minced and subtransplanted to new mice. Subtransplantation was repeated until a stable growing xenograft line after passage 3 was obtained. Pancreatic cancer spheres were generated as described recently ${ }^{31}$ and used for experiments between days 7 and 30 in culture. Patient material was obtained under the approval of the ethical committee of the University of Heidelberg after written informed consent of patients. Diagnoses were established by conventional clinical and histological criteria according to the WHO. All surgical resections were indicated by principles and practice of oncological therapy.

Treatment of cells. Cabozantinib (XL184 99\%, Selleckchem, Houston, TX, USA) was dissolved in DMSO to a $20 \mathrm{~mm}$ stock solution. Gemcitabine (Fresenius Kabi, Bad Homburg, Germany) was diluted in cell culture medium to a $100 \mu \mathrm{m}$ stock.

Apoptosis measurement. Cells were stained with FITC-conjugated annexinV (BD Biosciences, Heidelberg, Germany) and externalization of phosphatidylserine, as well as the forward side scatter profile were identified by flow cytometry (FACScan, BD Biosciences). DNA fragmentation was detected by staining of fragmented DNA with propidium iodide buffer according to the Nicoletti method. ${ }^{32}$ 
Viability assay. Viability was measured using 3-(4,5-dimethylthiazol-2-y))-2, 5-diphenyltetrazolium bromide (MTT) as described previously. ${ }^{18}$

Colony-forming assay. Cells cultured were left untreated or were treated with Cabozantinib, Gemcitabine or both together. Seventy-two hours later cells were seeded in complete medium in 6-well tissue culture plates and colony-forming assays were performed as described previously. ${ }^{18}$

Spheroid assay. For formation of spheroids, cells were cultured in NeuroCult NS-A basal serum-free medium (human) (StemCell Technologies, Vancouver, BC, Canada) supplemented with $2 \mu \mathrm{g} / \mathrm{ml}$ Heparin (StemCell Technologies), $20 \mathrm{ng} / \mathrm{ml}$ hEGF (R\&D Systems, Wiesbaden-Nordenstadt, Germany), $10 \mathrm{ng} / \mathrm{ml}$ hFGF-b (PeproTech, Hamburg, Germany) and NeuroCult NS-A Proliferation Supplements (StemCell Technologies). Cells were seeded at low densities $\left(2 \times 10^{2}-1 \times\right.$ $\left.10^{3} \mathrm{cell} / \mathrm{s} / \mathrm{ml}\right)$ in 12-well-low adhesion plates $(1 \mathrm{ml} / \mathrm{well})$.

Western blot analysis. Proteins were isolated, and western blot analysis was performed as described previously. ${ }^{18}$ The following antibodies were used: Mouse mAb against human XIAP (BD Biosciences, San Jose, CA, USA), rabbit mAb against human c-Met, SOX2, p53 (Cell Signaling, Danvers, MA, USA), Ki67 (Epitomics, Burlingame, CA, USA), rabbit pAb against human Bcl-2 (Cell Signaling), Bim (Abcam, Cambridge, UK), clAP2 (Epitomics), survivin and p-Met (R\&D Systems, Minneapolis, MN, USA)

LightCycler RT-PCR. Quantitative LightCycler-PCR was performed using the First Strand cDNA Synthesis Kit for RT-PCR LightCycler, FastStart DNA Master SYBR Green I (Roche Diagnostics GmbH, Mannheim, Germany) and a LightCycler-PCR machine (Roche), as described recently. ${ }^{33}$ Specific primer pairs (MWG Biotech, Ebersberg, Germany) for human bcl-2, survivin and sox2 were used and are available upon request.

Immunohistochemistry. Endogenous biotin was blocked using the Avidin/ Biotin blocking kit (Vector, Burlingame, CA, USA) according to the instructions of the manufacturer. Endogenous peroxidase was quenched by $0.3 \%$ in methanol. Biotinylated goat anti-rabbit IgG (Vector) was used as a secondary Ab. The signal was amplified using the ABC Elite kit (Vector). AEC was used as a chromogen. Samples were counterstained with hematoxylin (Mayer, Sigma, Taufkirchen, Germany), mounted in Pro Tags Aqua mount (Quartett, Berlin, Germany). Omission of the primary Ab served as a negative control. The signal was detected at $\times 400$ magnification using a Leica DMRB fluorescence microscope (Leica, Wetzlar; Germany). Images of representative fields were captured using a SPOT FLEX 15.2 64Mp shifting pixel digital color camera (Diagnostic Instruments Inc., Sterling Heights, MI, USA) and analyzed with SPOT Basic/Advanced 4.6 software (Diagnostic Instruments). Primary antibodies were: rabbit pAb anti human c-Met, CD133, SOX2 (Abcam) and cleaved fragment of human caspase-3 (R\&D Systems, Abingdon, UK).

Antibody array. Nitrocellulose membranes to which capture antibodies have been spotted and reagents for detection were obtained as 'Human apoptosis array kit' and 'Human pluripotent stem cell antibody array kit' from R\&D Systems (Wiesbaden-Nordenstadt). According to the manufacturer's instructions, protein extracts were prepared, incubated with the nitrocellulose membranes, followed by detection of specific protein binding with biotinylated secondary antibodies using streptavidin-HRP and chemiluminescence detection reagents.

Statistical analysis. MTT-assays, FACS-analysis, western blot analysis, spheroid- and colony-forming assays with established cell lines were repeated three times. GRT-PCR, protein array and staining of spheroidal cells from patient tumors were repeated only once due to limitation of sample availability (individual treatment rounds for long-time cabozantinib treatment, patient-derived cells) or high costs of experiments (antibody protein array). However, data of experiments, which were performed only once, were confirmed by the other data of the manuscript. The quantitative data are presented as the mean \pm S.D. The data were analyzed using Student's $t$-test for statistical significance. $P<0.05$ was deemed to be statistically significant.

\section{Conflict of Interest}

The authors declare no conflict of interest.
Acknowledgements. We thank L Disch, M Meinhardt, Sonja Bauer and Esther Soyka for excellent technical assistance. This study was supported by grants from the German Cancer Aid (Deutsche Krebshilfe 109362), German Research Community (DFG HE 3186/11-1), German-Israeli Foundation for Scientific Research and Development (GIF 1058-7.11/2008), Heidelberger Stiftung Chirurgie and from the Foundation for cancer and scarlet fever of the University of Heidelberg.

\section{Author's contribution}

$\mathrm{IH}$ : concept and design. $\mathrm{CH}, \mathrm{NG}, \mathrm{TG}$ : acquisition of data. $\mathrm{CH}, \mathrm{IH}$ : analysis and interpretation of data. $\mathrm{IH}, \mathrm{CH}$ : writing, review and/or revision of the manuscript. VR, FS, SL, CN, JM, JG, MWB: administrative, technical or material support

1. Gukovskaya AS, Pandol SJ. Cell death pathways in pancreatitis and pancreatic cancer. Pancreatology 2004; 4: 567-586.

2. Oberstein PE, Saif MW. First-line treatment for advanced pancreatic cancer. Highlights from the '2011 ASCO Gastrointestinal Cancers Symposium'. San Francisco, CA, USA. January 20-22, 2011. JOP 2011; 12: 96-100.

3. Conroy T, Desseigne F, Ychou M, Bouche O, Guimbaud R, Becouarn $Y$ et al. FOLFIRINOX versus gemcitabine for metastatic pancreatic cancer. N Engl J Med 2011; 364: 1817-1825.

4. Provenzano PP, Hingorani SR. Hyaluronan, fluid pressure, and stromal resistance in pancreas cancer. Br J Cancer 2013; 108: 1-8.

5. Rasheed ZA, Kowalski J, Smith BD, Matsui W. Concise review: emerging concepts in clinical targeting of cancer stem cells. Stem Cells 2011; 29: 883-887.

6. Lapidot T, Sirard C, Vormoor J, Murdoch B, Hoang T, Caceres-Cortes J et al. A cell initiating human acute myeloid leukaemia after transplantation into SCID mice. Nature 1994; 367: 645-648.

7. Al-Haij M, Wicha MS, Benito-Hernandez A, Morrison SJ, Clarke MF. Prospective identification of tumorigenic breast cancer cells. Proc Natl Acad Sci USA 2003; 100: 3983-3988.

8. Singh SK, Hawkins C, Clarke ID, Squire JA, Bayani J, Hide T et al. Identification of human brain tumour initiating cells. Nature 2004; 432: 396-401.

9. Simeone DM. Pancreatic cancer stem cells: implications for the treatment of pancreatic cancer. Clin Cancer Res 2008; 14: 5646-5648.

10. Li C, Heidt DG, Dalerba P, Burant CF, Zhang L, Adsay V et al. Identification of pancreatic cancer stem cells. Cancer Res 2007; 67: 1030-1037.

11. Hermann PC, Huber SL, Herrler T, Aicher A, Ellwart JW, Guba M et al. Distinct populations of cancer stem cells determine tumor growth and metastatic activity in human pancreatic cancer. Cell Stem Cell 2007; 1: 313-323.

12. Li C, Wu JJ, Hynes M, Dosch J, Sarkar B, Welling TH et al. c-Met is a marker of pancreatic cancer stem cells and therapeutic target. Gastroenterology 2011; 141: 2218-2227.e5.

13. Gherardi E, Birchmeier W, Birchmeier C, Vande Woude G. Targeting MET in cancer: rationale and progress. Nat Rev Cancer 2012; 12: 89-103.

14. Li Y, Li A, Glas M, Lal B, Ying M, Sang Y et al. c-Met signaling induces a reprogramming network and supports the glioblastoma stem-like phenotype. Proc Natl Acad Sci USA 2011; 108: $9951-9956$

15. You WK, Sennino B, Williamson CW, Falcon B, Hashizume H, Yao LC et al. VEGF and $\mathrm{c}-$ Met blockade amplify angiogenesis inhibition in pancreatic islet cancer. Cancer Res 2011; 71: 4758-4768.

16. Ryan DP. An open-label, phase II study of cabozantinib (XL184) in advanced pancreatic neuroendocrine and carcinoid tumors. Clinical Trials 2011; ClinicalTrials.gov Identifier: NCT01466036 Study ID Number: 11-274.

17. U.S.Food and Drug Administration: Cabozantinib 2013.

18. Kallifatidis G, Rausch V, Baumann B, Apel A, Beckermann BM, Groth A et al. Sulforaphane targets pancreatic tumour-initiating cells by NF-kappaB-induced antiapoptotic signalling. Gut 2009; 58: 949-963.

19. Abbott A. Cancer: the root of the problem. Nature 2006; 442: 742-743.

20. Flores ER, Lozano G. The p53 family grows old. Genes Dev 2012; 26: 1997-2000.

21. Takahashi K, Tanabe K, Ohnuki M, Narita M, Ichisaka T, Tomoda K et al. Induction of pluripotent stem cells from adult human fibroblasts by defined factors. Cell 2007; 131 : 861-872.

22. Krizhanovsky V, Lowe SW. Stem cells: the promises and perils of p53. Nature 2009; 460 : 1085-1086.

23. Song $Y$, Washington MK, Crawford HC. Loss of FOXA1/2 is essential for the epithelial-tomesenchymal transition in pancreatic cancer. Cancer Res 2010; 70: 2115-2125.

24. Suzuki $A$, Nakauchi $H$, Taniguchi $H$. Prospective isolation of multipotent pancreatic progenitors using flow-cytometric cell sorting. Diabetes 2004; 53: 2143-2152.

25. Oshima Y, Suzuki A, Kawashimo K, Ishikawa M, Ohkohchi N, Taniguchi H. Isolation of mouse pancreatic ductal progenitor cells expressing CD133 and c-Met by flow cytometric cell sorting. Gastroenterology 2007; 132: 720-732.

26. Birchmeier C, Birchmeier W, Gherardi E, Vande Woude GF. Met, metastasis, motility and more. Nat Rev Mol Cell Biol 2003; 4: 915-925.

27. Cometriq (cabozantinib capsules) prescribing information. Exelixis 2012.

28. Niwa $\mathrm{H}$, Ogawa $\mathrm{K}$, Shimosato $\mathrm{D}$, Adachi $\mathrm{K}$. A parallel circuit of LIF signalling pathways maintains pluripotency of mouse ES cells. Nature 2009; 460: 118-122. 
29. Valeriote F, van Putten L. Proliferation-dependent cytotoxicity of anticancer agents: a review. Cancer Res 1975; 35: 2619-2630.

30. Rausch V, Liu L, Kallifatidis G, Baumann B, Mattern J, Gladkich J et al. Synergistic activity of sorafenib and sulforaphane abolishes pancreatic cancer stem cell characteristics. Cancer Res 2010; 70: 5004-5013.

31. Lonardo E, Hermann PC, Mueller MT, Huber S, Balic A, Miranda-Lorenzo I et al. Nodal/ Activin signaling drives self-renewal and tumorigenicity of pancreatic cancer stem cells and provides a target for combined drug therapy. Cell Stem Cell 2011; 9: 433-446.

32. Nicoletti I, Migliorati G, Pagliacci MC, Grignani F, Riccardi C. A rapid and simple method for measuring thymocyte apoptosis by propidium iodide staining and flow cytometry. J Immunol Methods 1991; 139: 271-279.
33. Rausch V, Liu L, Apel A, Rettig T, Gladkich J, Labsch S et al. Autophagy mediates survival of pancreatic tumour-initiating cells in a hypoxic microenvironment. J Pathol 2012; 227 325-335.

cc) (\$) $\ominus$ Cell Death and Disease is an open-access journal published by Nature Publishing Group. This work is licensed under a Creative Commons Attribution-NonCommercialNoDerivs 3.0 Unported License. To view a copy of this license, visit http://creativecommons.org/licenses/by-nc-nd/3.0/

Supplementary Information accompanies this paper on Cell Death and Disease website (http://www.nature.com/cddis) 